\title{
Trends in the neurodevelopmental outcomes among preterm infants from 2003-2012: a retrospective cohort study in Japan
}

\author{
Hidehiko Nakanishi ${ }^{1} \cdot$ Hideyo Suenaga ${ }^{1}$ - Atsushi Uchiyama ${ }^{1} \cdot$ Yumi Kono $^{2}$ • \\ Satoshi Kusuda ${ }^{3}$ On behalf of the Neonatal Research Network, Japan
}

Received: 16 August 2017 / Revised: 10 December 2017 / Accepted: 22 January 2018 / Published online: 20 April 2018

(c) The Author(s) 2018. This article is published with open access

\begin{abstract}
Objectives To determine the trends in mortality and the prevalence of abnormal neurodevelopmental outcomes among preterm Japanese infants.

Study design A retrospective multicenter cohort of 30,793 preterm infants born at a gestational age $\leq 32$ weeks, between 2003 and 2012, in the Neonatal Research Network, Japan, was evaluated in the primary analysis. Finally, 13,661 infants were followed-up until 3 years of age and evaluated for neurodevelopmental outcomes, including cerebral palsy (CP), home oxygen therapy (HOT) use, and visual, hearing, and cognitive impairments. Multivariable logistic regression analysis was performed to determine the risk-adjusted trends in mortality and long-term neurodevelopmental outcomes.

Results The trends in overall mortality (adjusted odds ratio, (AOR): 0.92; 95\% confidence interval, (CI): 0.89-0.94), the prevalence of CP (AOR: 0.95, 95\% CI: 0.92-0.98), HOT use (AOR: 0.84, 95\% CI: 0.75-0.93), and visual (AOR: 0.84, 95\% CI: 0.81-0.87) and hearing impairments (AOR: 0.78, 95\% CI: 0.63-0.97) showed a significant downward trend, while cognitive impairment showed no significant changes (AOR: 1.02, 95\% CI: 0.99-1.05). Intravenous hyperalimentation was significantly correlated with visual impairment (AOR 0.74, 95\% CI 0.59-0.91). Early establishment of enteral feeding was associated with improved long-term outcomes.

Conclusions Mortality was improved, and this did not lead to increased risks for abnormal neurodevelopmental outcomes. Nutritional support might improve long-term neurodevelopmental outcomes.
\end{abstract}

\section{Introduction}

While advances in perinatal and neonatal care management have led to increased survival rates among premature infants, worldwide [1-5], there have been concerns that the declining mortality may lead to an increase in the number of surviving infants with neurodevelopmental impairments. A recently conducted multicenter, multinational cohort of

Electronic supplementary material The online version of this article (https://doi.org/10.1038/s41372-018-0061-7) contains supplementary material, which is available to authorized users.

\footnotetext{
Hidehiko Nakanishi

hidehiko@qf6.so-net.ne.jp

1 Maternal and Perinatal Center, Tokyo Women's Medical University, Tokyo, Japan

2 Department of Pediatrics, Jichi Medical University, Tochigi, Japan

3 Department of Pediatrics, Kyorin University, Tokyo, Japan
}

preterm infants among eight members of the International Network for Evaluating Outcomes, spanning nine countries, found that Japan had the lowest mortality [6]. However, few studies have examined the mortality and long-term neurodevelopmental outcomes among surviving premature infants using a large cohort. The findings of such studies would be important for clinicians, both in counseling and early-care decision-making for these high-risk infants. Furthermore, these findings might also be useful in identifying outcome variations in premature infants, across countries [6-8].

Therefore, the aim of our study was to characterize the recent trends in morbidity, mortality, and long-term neurodevelopmental outcomes, among preterm infants born at a gestational age (GA) $\leq 32$ weeks, between 2003 and 2012, using data from the Neonatal Research Network, Japan (NRNJ) [9]. Furthermore, we also explored potential interventions associated with long-term neurodevelopmental outcomes. 


\section{Materials and methods}

\section{Study design and population}

This retrospective multicenter cohort study was carried out between 2003 and 2012. The 202 participating hospitals included large tertiary perinatal centers, designated as tertiary neonatal intensive care units (NICU), in Japan. The central internal review board at Tokyo Women's Medical University approved this study. Written informed consent was obtained from the parents or guardians of the participants. Data were collected anonymously, and unlinked from individual data before analysis.

Of the total 40,806 infants with a birth weight $\leq 1500$ g, 10,013 cases (with congenital anomaly, missing medical records, and births outside hospitals) [10] and 155 cases of death in the delivery room were excluded. The final study population of 30,638 infants with a GA $\leq 32$ weeks were then admitted to the NICU, and grouped into four GA categories $(22-24,25-27,28-30$, and 31-32 weeks). The characteristics of the participating hospitals over time are shown in Supplementary Table 1. To evaluate the neurodevelopmental outcomes among these infants, 13,661 infants with follow-up data at 3 years of age (from 132 tertiary perinatal centers) were analyzed while 14,971 infants (deaths at and after discharge, and those lost to follow-up by 3 years of age) were excluded (Fig. 1). The differences in variables between infants with and without follow-up data $(13,661$ vs. 14,971, respectively) are shown in Supplementary Tables 2 and 3.

\section{Definitions}

All study parameters were defined based on the NRNJ registration manual [2,9]. Data on GA were obtained from obstetric histories with confirmation or corrections using ultrasound examination at health check-ups for pregnant women during the first trimester. Congenital anomaly was defined as a major life-threatening anomaly that did not include just external malformations. The study outcomes included neonatal outcomes (mortality and survival) and long-term neurodevelopmental outcomes at 3 years of age, such as the prevalence of cerebral palsy (CP) [11], home oxygen therapy (HOT) use, and visual, hearing, and cognitive impairments, as indicated by developmental testing, according to the Kyoto Scale of Psychological Development (KSPD) [12, 13]. Mortality was defined as death occurring among surviving in-born infants (born at any of the participating perinatal centers with no transfer to other hospitals after birth) admitted to the NICU, before discharge (deaths in the delivery room were excluded). CP was defined at 3 years of age at any level of severity, as reported by Bax [14]. Visual impairment was defined as blindness with no functional vision in one or both eyes. Hearing impairment was considered present when amplification was
Fig. 1 Flow chart of the evaluated infants. Of the 40,806 infants registered to the participating neonatal centers in the NRNJ, from 2003 to 2012 , 30,638 infants admitted to the NICU were categorized into the following four groups: $22-24$, 25-27, 28-30, and

31-32 weeks' GA. Furthermore, 13,661 infants, followed up until 3 years of age, were evaluated for long-term outcomes. NICU neonatal intensive care unit, NRNJ Neonatal Research Network, Japan, GA gestational age, DR delivery room

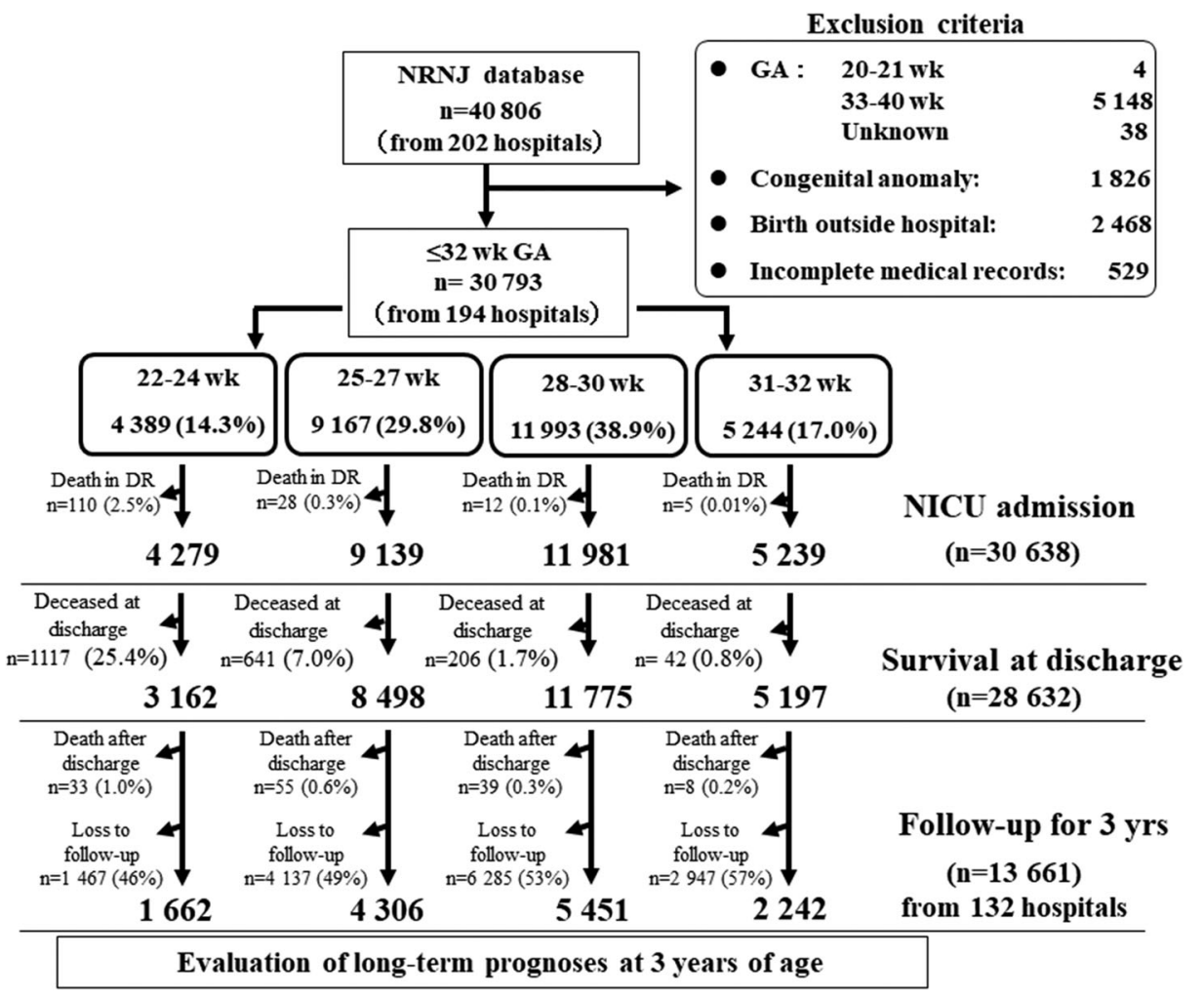


required. A total Developmental Quotient score $<70$, equivalent to a Bayley III Cognitive Scale score $<85$, represented significantly delayed performance [13].

Trends in mortality and neurodevelopmental outcomes, by GA categories as percentages, were determined for the study participants, over the study period. With a low response rate $(<60 \%$ from 2003 to 2005) in terms of HOT use, at 3 years of age in the NRNJ, the trends in HOT use were determined from 2006 to 2012.

Data were obtained on the antenatal characteristics of the participants, including maternal age, parity, multiple pregnancies, pregnancy-induced hypertension (PIH), maternal diabetic mellitus (DM), premature rupture of membrane (PROM), clinical chorioamnionitis (CAM), non-reassuring fetus status (NRFS), head presentation, antenatal steroid (ANS) use, and cesarean section. Data on the neonatal characteristics, including GA, birth weight, male sex, small for GA (SGA), and 1-minute Apgar score were also obtained. Furthermore, information on neonatal morbidities including the 5-minutes Apgar score, respiratory distress syndrome (RDS), pulmonary hemorrhage, air-leak syndrome, persistent pulmonary hypertension of the newborn (PPHN), patent ductus arteriosus (PDA), bronchopulmonary dysplasia (BPD) at 36 weeks [15], adrenal insufficiency of prematurity (AOP), intraventricular hemorrhage (IVH), retinopathy of prematurity (ROP) [16], periventricular leukomalacia (PVL), sepsis, and necrotizing enterocolitis/interstitial perforation (NEC/IP) was obtained from each perinatal center's records. Data on the different interventions (intubation, mechanical ventilation, surfactant administration, high frequency oscillatory ventilation (HFOV), inhaled nitric oxide (iNO), steroids for BPD, indomethacin for PDA, PDA ligation, intravenous hyperalimentation, ROP treatment, time to establishment of enteral feeding (defined as the number of days till enteral feeding of $100 \mathrm{ml} / \mathrm{kg} /$ day was achieved), and HOT use at discharge were also obtained from each perinatal center's records.

\section{Statistical analyses}

Statistical analyses were conducted using JMP ${ }^{\circledR} 13$ (SAS Institute Inc., Cary, NC, USA). Data were reported using the mean (continuous data) or the percentage (categorical data) and $95 \%$ confidence interval $(95 \% \mathrm{CI})$. Variables with a non-normal distribution were expressed using median and interquartile range (IQR), and analyzed using Wilcoxon rank sum tests or Kruskal-Wallis one-way analysis of variance. The $\chi^{2}$-test was performed for yearly trends in proportions (Cochran-Armitage test) over the past decade, as well as for the first (2003-2007) and second 5-year period (2008-2012) for categorical data (antenatal and neonatal correlates, morbidity, interventions, mortality, and long-term neurodevelopmental outcomes).

We examined the risk-adjusted trends in morbidity, intervention, mortality and long-term neurodevelopmental outcomes, over time, by adjusting for patients' background characteristics and related perinatal (antenatal and neonatal) risk factors; this is because, with the number of participating hospitals in the NRNJ increasing, annually, clinical conditions may change over time. To avoid bias among the registered hospitals in NRNJ, infants from the 38 originally participating hospitals, which were constantly tracked over the entire study period, were chosen.

Three levels of analysis were performed, as follows: the significant independent perinatal risk factors for mortality were determined using univariate logistic regression analysis (Analysis \#1) among all the participating infants; multiple logistic regression analysis (Analysis \#2) was performed to determine the risk-adjusted trends by adjusting for year and those significant perinatal factors determined in Analysis \#1; multivariable logistic analysis (Analysis \#3) was performed to determine the interventions associated with long-term neurodevelopmental outcomes by adjusting for year and the perinatal risk factors identified in Analysis \#1. A $p$-value $<0.05$ was considered significant. The odds ratios (ORs) with $95 \%$ CIs were reported.

\section{Results}

A majority of the participants $(99.5 \%)$ were admitted to the NICU soon after birth, with no differences across GA categories in the admission rates (Supplementary Table 4). The lowest GA group (22-24 weeks) had the highest intubation rate (93.2\% [95\% CI: 92.3-93.9]). The total mortality for all the participants was $6.5 \%$, with a dramatic decrease, by GA category, from $26.1 \%$ (22-24 weeks) to $0.8 \%$ (31-32 weeks).

The trends in mortality and the prevalence of neurodevelopmental outcomes are shown in Fig. 2. A significant decrease in the overall trend (total) in mortality was observed, over time (from $9.0 \%$ in 2003 to $4.6 \%$ in 2012), and by GA categories (Fig. 2a). The overall prevalence of CP (from $12.3 \%$ in 2003 to $7.1 \%$ in 2012), HOT use (2.4\% in 2006 to $0.9 \%$ in 2012), and visual impairment (11.4\% in 2003 to $3.2 \%$ in 2012) showed a downward trend, over time. In the GA categories, similar patterns were shown for $\mathrm{CP}$ and visual impairment, but not HOT use (Fig. 2b-d). In the second 5-year period, among those with a GA of 22-24 weeks, the prevalence of CP showed an upward trend (Fig. 2b). Although no significant changes were observed for both hearing and cognitive impairments, over time (Fig. 2e, f), a significant decrease from $1.8 \%$ in 2008 to 


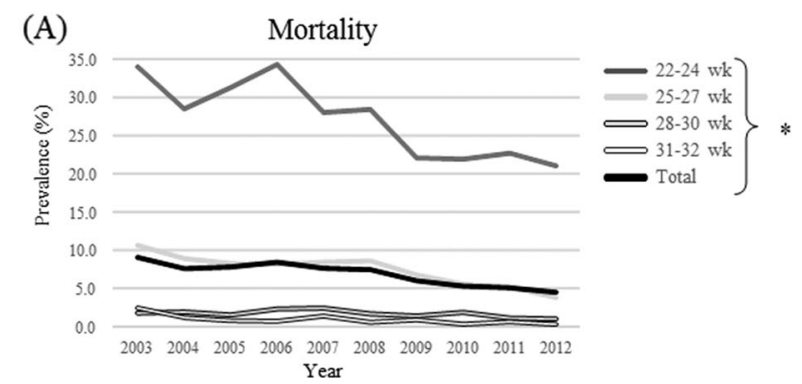

(C) Home oxygen therapy use
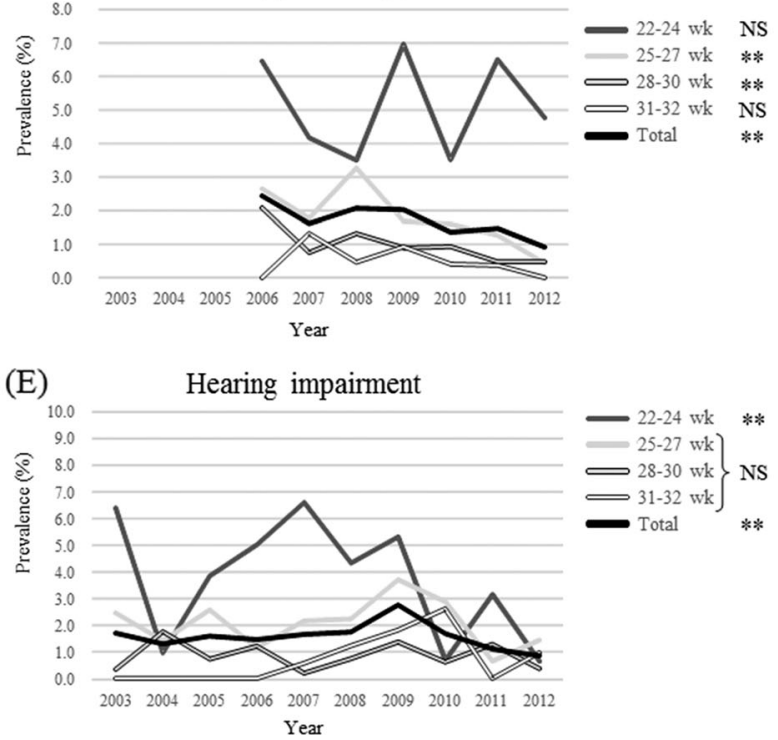

* Significant decreasing tendency with $\mathrm{p}<0.05$ over the past decade NS, not significant

Fig. 2 Trends in a mortality and neurodevelopmental outcomes at 3 years of age, $\mathbf{b}$ CP, $\mathbf{c}$ HOT, $\mathbf{d}$ visual impairment, $\mathbf{e}$ hearing impairment, f cognitive impairment, by GA among all infants. $p$-Values were

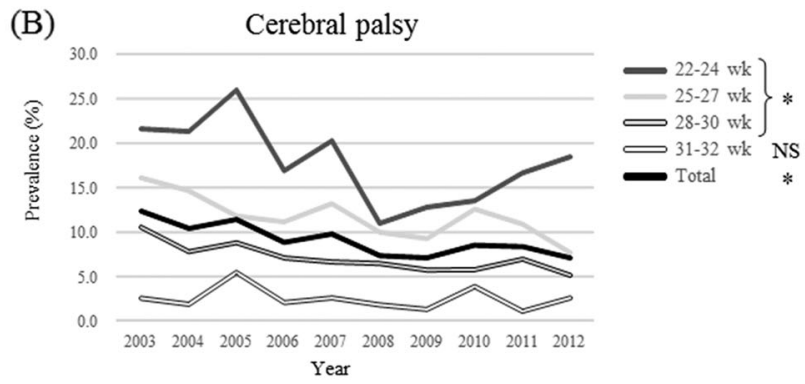

(D)

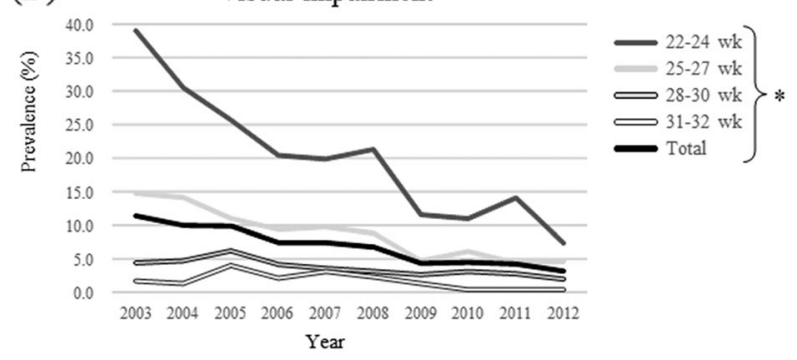

(F)

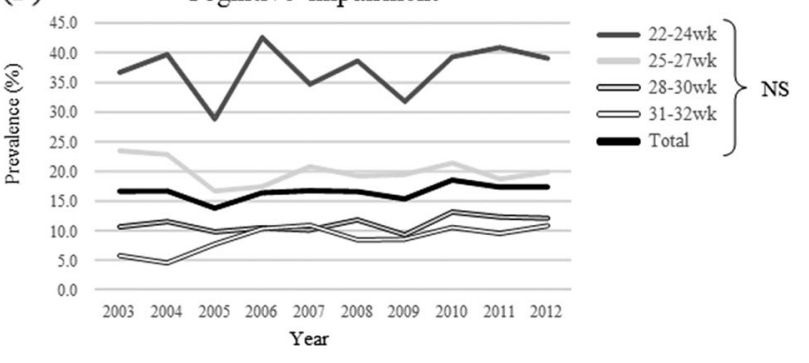

** Significant decreasing tendency with $\mathrm{p}<0.05$ in the second five-year period

calculated by the Cochrane-Armitage $\chi^{2}$-test. *Significant decrease in 2003-2012; **significant decrease in 2008-2012; NS not significant. CP cerebral palsy, HOT home oxygen therapy, GA gestational age

onset sepsis as well as NEC and/or IP, especially in the first 5year period. For severe IVH, severe ROP, and PVL, a significant decrease in prevalence was shown, over time. Of the interventions (Table 2), intubation after birth, PDA ligation, HFOV, surfactant administration, indomethacin for PDA, ROP treatment, HOT use at discharge, and intravenous hyperalimentation showed an upward trend, over time. However, in the case of steroid treatment for BPD, a downward trend was shown, over time, especially in the second 5year period.

In Analysis \#1, where the independent perinatal risk factors of mortality were assessed (not shown), GA (adjusted odds ratio (AOR) $0.77,95 \%$ CI $0.73-0.81$ ), birth weight at increments of $100 \mathrm{~g}$ (AOR $0.74,95 \% \mathrm{CI}$ 0.71-0.78), male sex (AOR 1.37, 95\% CI 1.23-1.52), Apgar score $<4$ at $1 \mathrm{~min}$ (AOR 2.24, 95\% CI 2.01-2.51), multiple pregnancies (AOR 1.34, 95\% CI 1.18-1.53), ANS (AOR 0.63, 95\% CI 0.57-0.71), PIH (AOR 0.71, 95\% CI 


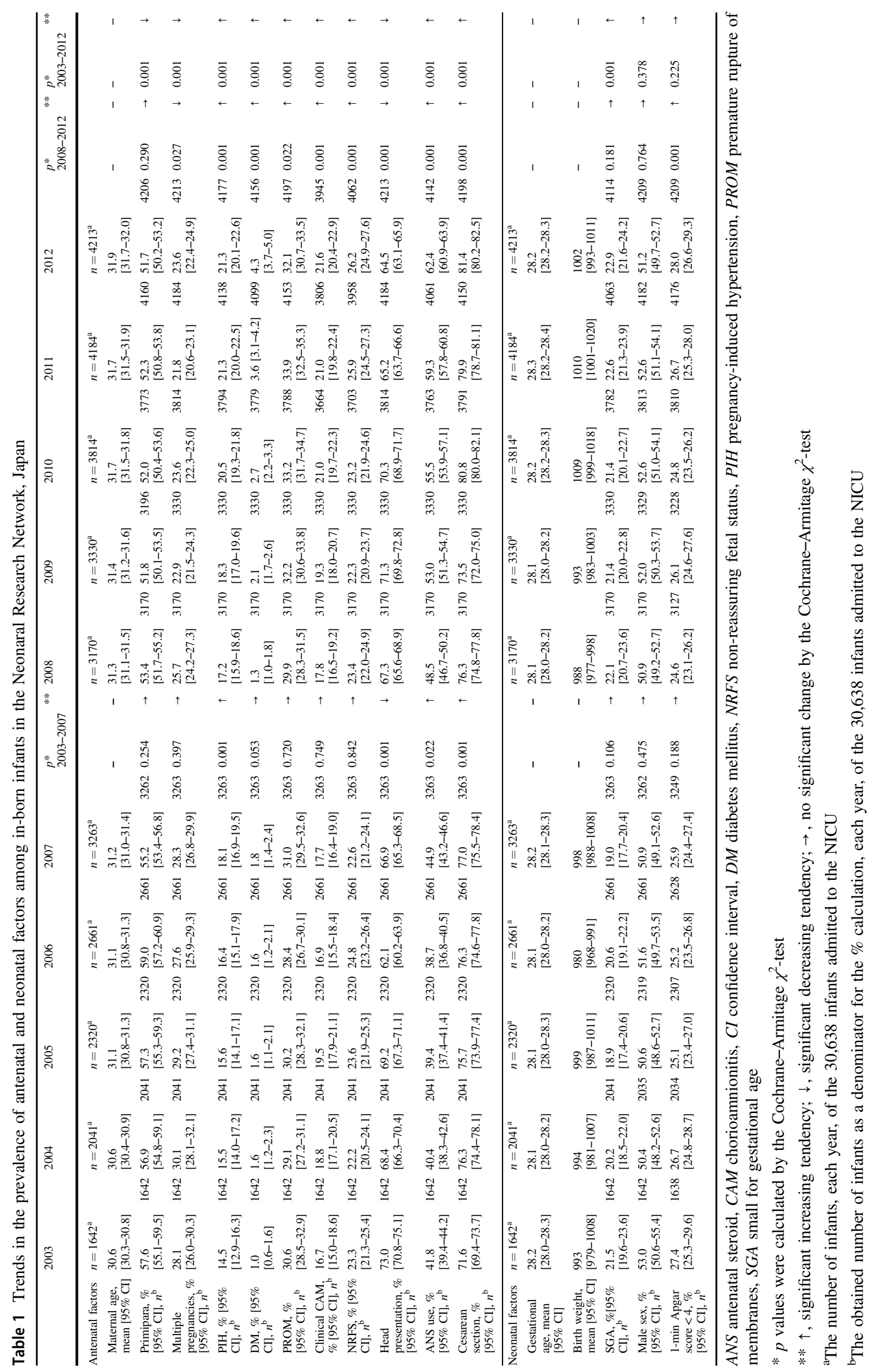




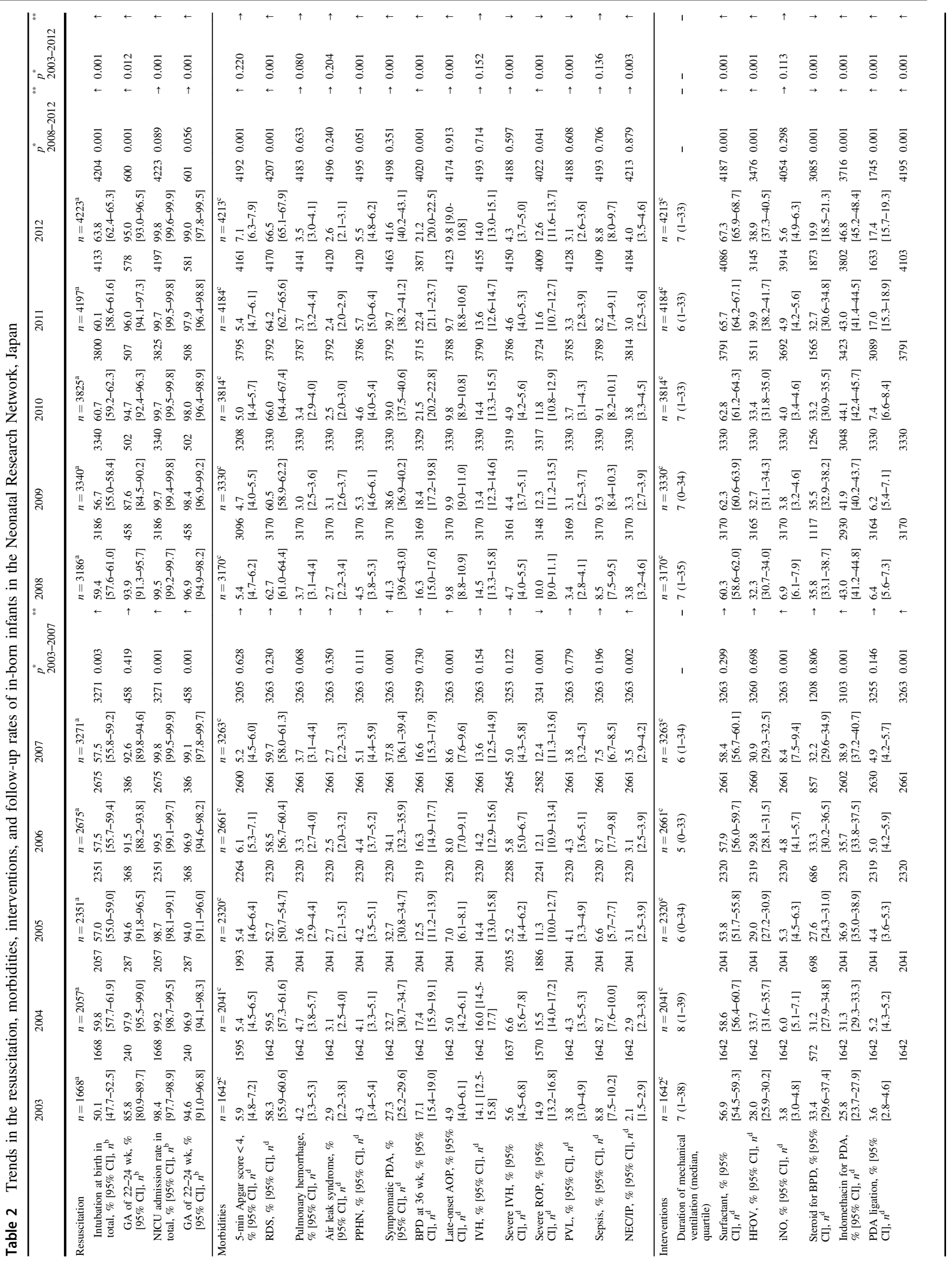




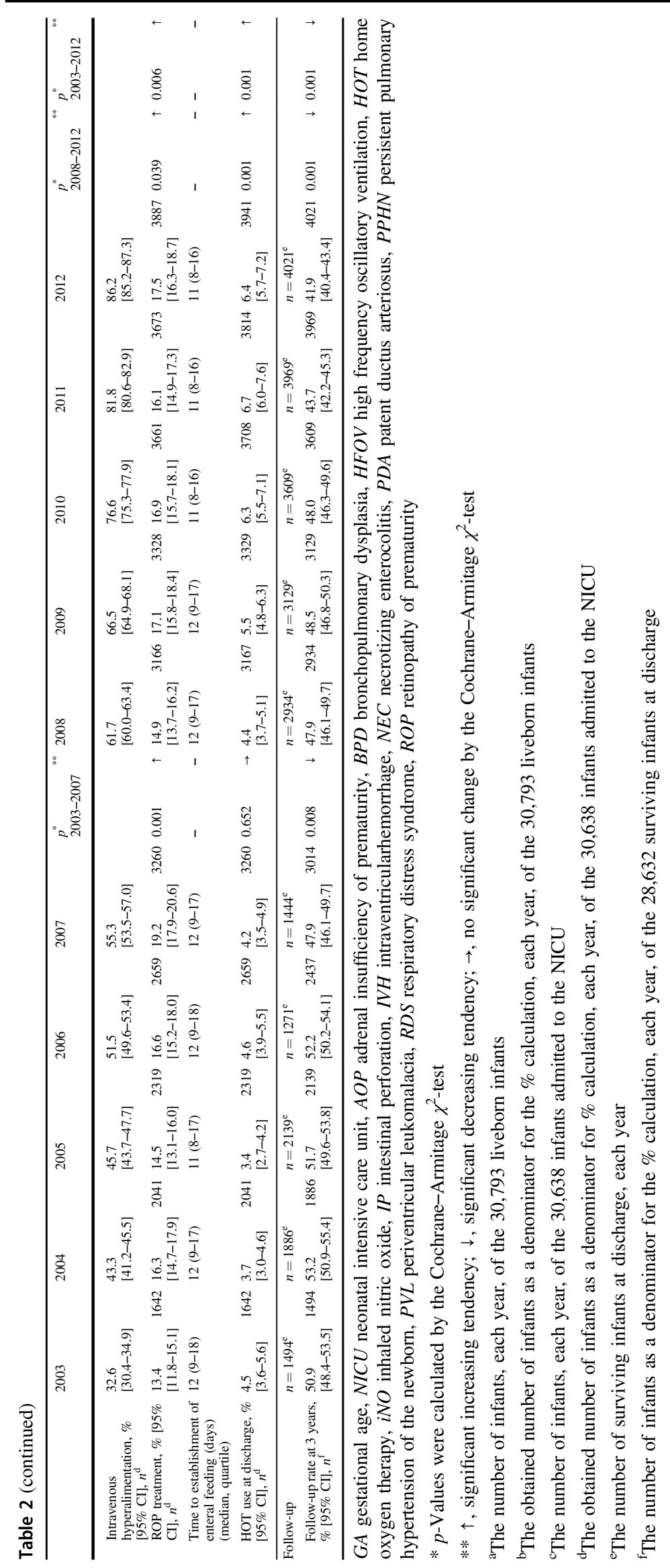


0.59-0.85), clinical CAM (AOR 0.84, 95\% CI 0.74-0.96), NRFS (AOR 1.54, 95\% CI 1.37-1.74), and cesarean section (AOR 0.82, 95\% CI 0.73-0.94) remained independent predictors of mortality. These factors adjusted for year, were then used to determine the risk-adjusted trends, over time.

As shown in Table 3 (Analysis \#2), over time, in the riskadjusted trends in the prevalence of morbidities, the significant upward trend persisted for RDS, PPHN, BPD (28 days and 36 weeks), PDA, late-onset AOP, and NEC/ IP, and a significant decrease was noted for severe IVH, severe ROP and PVL. In terms of interventions, over time, the significant upward trend persisted for HFOV, surfactant administration, indomethacin for PDA, PDA ligation, intravenous hyperalimentation, ROP treatment, and HOT use. Importantly, for mortality and long-term neurodevelopmental outcomes, over time, the significant downward trend persisted for mortality, the prevalence of $\mathrm{CP}$, HOT use, and visual and hearing impairment, while no significant changes were noted for cognitive impairment.

As shown in Table 4 (Analysis \#3), in the adjusted model, nutritional support remained correlated with longterm neurodevelopmental outcomes with intravenous hyperalimentation remaining an independent correlate of visual impairment (AOR 0.74, 95\% CI 0.59-0.91). Furthermore, the AOR of time to establishment of enteral feeding (with 5-day increments) for all disabilities suggested that the shorter the time to the establishment of full enteral feeding, the lower the prevalence of abnormal longterm neurodevelopmental outcomes (Table 4).

\section{Discussion}

An important contribution of our study is that it elucidated the trends, over time, in the prevalence of abnormal longterm neurodevelopmental outcomes, among in-born preterm infants, excluding births occurring outside the participating hospitals, which were strongly associated with the survival of extremely preterm infants [10]. As shown in Supplementary Table 1, in accordance with increased number of participating hospitals, the number of infants increased: however the average number of infants per hospital decreased. This suggests that more hospitals with low numbers of NICU beds had gradually been increased in the NRNJ, over the study period. As the NRNJ database covered almost $70 \%$ of all nationally delivered preterm infants with a birth weight $\leq 1500 \mathrm{~g}$, in 2012 , our study seems to correspond to a nationwide survey of premature infants in Japan.

We investigated the recent trends in mortality and abnormal neurodevelopmental outcomes, and demonstrated that the increased survival of preterm infants was not associated with a concomitant increase in long-term
Table 3 Risk-adjusted trends in the morbidities, intervention, mortality, and long-term neurodevelopmental outcomes of the inborn patients from the 38 originally participating hospitals

\begin{tabular}{lcr}
\hline & AOR & 95\% CI \\
\hline Morbidities & & \\
RDS & 1.05 & $1.03-1.06$ \\
PPHN & 1.03 & $1.01-1.06$ \\
Symptomatic PDA & 1.06 & $1.05-1.08$ \\
BPD at 36 wk & 1.05 & $1.03-1.07$ \\
Late-onset AOP & 1.08 & $1.06-1.11$ \\
Severe IVH & 0.96 & $0.93-0.99$ \\
Severe ROP & 0.97 & $0.96-0.99$ \\
PVL & 0.95 & $0.92-0.98$ \\
NEC/interstitial perforation & 1.04 & $1.01-1.08$ \\
Intervention & & \\
Surfactant & 1.06 & $1.05-1.07$ \\
HFOV & 1.06 & $1.04-1.07$ \\
iNO & 1.02 & $0.99-1.05$ \\
Indomethacin for PDA & 1.12 & $1.10-1.13$ \\
PDA ligation & 1.16 & $1.13-1.20$ \\
Steroid for BPD & 0.99 & $0.97-1.01$ \\
Intravenous hyperalimentation & 1.31 & $1.30-1.33$ \\
ROP treatment & 1.02 & $1.00-1.04$ \\
HOT use at discharge & 1.10 & $1.07-1.13$ \\
Mortality & & \\
Deceased at discharge & 0.92 & $0.89-0.94$ \\
Long-term neurodevelopmental outcomes at 3 years of age \\
HOT use* & 0.95 & $0.92-0.98$ \\
Visual impairment & 0.84 & $0.76-0.94$ \\
Hearing impairment* & 0.84 & $0.81-0.87$ \\
Cognitive impairment & 0.79 & $0.63-0.97$ \\
\hline Year gestatonat & 1.02 & $0.99-1.05$ \\
\hline
\end{tabular}

Year, gestational age, birthweight, male sex, Apgar score $<4$ at $1 \mathrm{~min}$, multiple pregnancy, antenatal steroid use, pregnancy induced hypertension, clinical chorioamnionitis, non-reassuring fetus status, and cesarean section were used to adjust for changes in the background risks, over time

$A O R$ adjusted odds ratio, $A O P$ adrenal insufficiency of prematurity, $B P D$ bronchopulmonary dysplasia, $C I$ confidence interval, $C P$ cerebral palsy, $D Q$ developmental quotient, $H F O V$ high frequency oscillatory ventilation, $H O T$ home oxygen therapy, $i N O$ inhaled nitric oxide, $I V H$ intraventricular hemorrhage, $N E C$ necrotizing enterocolitis, $P D A$ patent ductus arteriosus, $P P H N$ persistent pulmonary hypertension of the newborn, $P V L$ periventricular leukomalacia, $R D S$ respiratory distress syndrome, $R O P$ retinopathy of prematurity

* AOR was calculated among patients in the second 5-year period, 2008-2012

disabilities. However, there were concerns over some of the GA categories. For example, the prevalence of HOT use at 3 years of age, in the 22-24 weeks GA group, showed no downward trend. It is thought that preterm infants with a GA of 22-24 weeks, originally born with an 
Table 4 Interventions associated with long-term neurodevelopmental outcomes at 3 years of age among the in-born infants from the original 38 participating hospitals over time

\begin{tabular}{|c|c|c|c|c|c|c|c|c|c|c|}
\hline \multirow[t]{2}{*}{ Intervention } & \multicolumn{2}{|l|}{$\mathrm{CP}$} & \multicolumn{2}{|c|}{ Visual impairment } & \multicolumn{2}{|c|}{$\begin{array}{l}\text { Hearing } \\
\text { impairment } \\
2008-2012 * *\end{array}$} & \multicolumn{2}{|c|}{$\begin{array}{l}\text { HOT } \\
2008-2012 * * *\end{array}$} & \multicolumn{2}{|c|}{$\begin{array}{l}\text { Cognitive } \\
\text { impairment }\end{array}$} \\
\hline & $\mathrm{AOR}^{*}$ & $95 \% \mathrm{CI}$ & $\mathrm{AOR}^{*}$ & $95 \% \mathrm{CI}$ & $\mathrm{AOR}^{*}$ & $95 \% \mathrm{CI}$ & $\mathrm{AOR}^{*}$ & $95 \% \mathrm{CI}$ & $\mathrm{AOR}^{*}$ & $95 \% \mathrm{CI}$ \\
\hline Surfactant & 1.69 & $1.39-2.07$ & 1.49 & $1.18-1.88$ & 1.75 & $0.78-3.92$ & 1.51 & $0.74-3.08$ & 1.13 & $0.96-1.33$ \\
\hline HFOV & 1.14 & $0.95-1.37$ & 1.18 & $0.95-1.45$ & 1.77 & $0.90-3.47$ & 1.76 & $0.95-3.26$ & 1.14 & $0.97-1.33$ \\
\hline iNO & 1.22 & $0.86-1.73$ & 1.18 & $0.80-1.76$ & 0.89 & $0.27-2.99$ & 2.28 & $1.13-4.57$ & 1.15 & $0.86-1.54$ \\
\hline Indomethacin & 1.31 & $1.09-1.57$ & 1.23 & $0.99-1.52$ & 1.71 & $0.86-3.40$ & 0.90 & $0.49-1.67$ & 1.15 & $0.99-1.34$ \\
\hline PDA ligation & 1.51 & $1.16-1.97$ & 1.53 & $1.15-2.03$ & 2.16 & $0.91-5.10$ & 1.81 & $0.91-3.59$ & 1.39 & $1.10-1.77$ \\
\hline Steroid for BPD & 1.09 & $0.86-1.39$ & 1.06 & $0.82-1.36$ & 0.63 & $0.27-1.47$ & 2.45 & $1.36-4.43$ & 1.38 & $0.90-1.33$ \\
\hline Intravenous hyperalimentation & 1.03 & $0.85-1.24$ & 0.74 & $0.59-0.91$ & 1.35 & $0.60-3.05$ & 1.17 & $0.57-2.43$ & 1.10 & $0.94-1.29$ \\
\hline $\begin{array}{l}\text { Time to establishment of enteral feeding } \\
\text { ( } 5 \text { days increment) }\end{array}$ & 1.13 & $1.09-1.16$ & 1.06 & $1.02-1.10$ & 1.11 & $1.04-1.18$ & 1.11 & $1.04-1.18$ & 1.09 & $1.06-1.13$ \\
\hline ROP treatment & 1.41 & $1.16-1.71$ & 3.73 & $3.03-4.59$ & 1.21 & $0.60-2.47$ & 1.34 & $0.77-2.36$ & 1.40 & $1.19-1.64$ \\
\hline
\end{tabular}

$A O R$ adjusted odds ratio, $B P D$ bronchopulmonary dysplasia, $C I$ confidence interval, $C P$ cerebral palsy, $H O T$ home oxygen therapy, $H F O V$ highfrequency oscillatory ventilation, $i N O$ inhaled nitric oxide, $P D A$ patent ductus arteriosus, $R O P$ retinopathy of prematurity

*Adjusted for year, gestational age, birth weight, male sex, Apgar score $<4$ at $1 \mathrm{~min}$, multiple pregnancy, antenatal steroid use, pregnancy induced hypertension, clinical chorioamnionitis, non-reassuring fetus status, and cesarean section

** Evaluated in the second half, 2008-2012, because trends in its prevalence showed decreasing tendency during those terms

*** Evaluated in the second half, 2008-2012, because there were lots of missing data, in the first half, 2003-2007

extremely premature lung structure and function, may not achieve the complete recovery of their lungs by 3 years of age. Hence, it is thought that it might be difficult to stop HOT use in these infants, by 3 years of age. The overall trend in the prevalence of cognitive impairment, which showed no change, over time, in this study, is similar to that observed in a report of periviable infants (GA of 22-24 weeks) with cognitive impairment in 2000-2011 [17]. This was thought to be due to the threshold of NICU admissions, especially for extremely premature infants. However, the absence of an increasing rate of disabilities, despite the increased number of surviving infants in this study, might reflect the advances in perinatal care in Japan. It is unknown why the trend in $\mathrm{CP}$, in the 22-24 weeks GA group, showed a significantly increasing tendency, especially in the second 5-year period. The prevalence of $\mathrm{CP}$ in our study was similar to that observed in another study which showed an increasing tendency [17]. Thus, further studies involving longer follow-up periods are needed among this group.

The findings of this study seem to support the notion that nutritional management is important for improved longterm neurodevelopmental outcomes among preterm infants $[18,19]$. Parenteral nutrition and early postnatal enteral feeding could help improve the development of gastrointestinal function, which might lead to a reduction in the time to full feeding commencement $[20,21]$. In our study, the time to establish full enteral feeding was inversely correlated with long-term neurodevelopmental disabilities, supporting the importance of the early establishment of enteral feeding for long-term neurodevelopmental outcomes. The induction of intravenous hyperalimentation was associated with visual impairment (AOR 0.73 95\% CI 0.59-0.90). Nutritional conditions including the levels of the systemic insulin-like growth factor 1 (IGF-1) are correlated with the pathogenesis of ROP [22]. Preterm infants with poor postnatal nutrition and growth have low circulating tissue concentrations of IGF-1 [23], which is associated with severe ROP [24].

The constant high rate of intubation after birth and NICU admission, among those with a GA of 22-24 weeks, might be one of the unique aspects of intensive care for extremely preterm infants, in Japan. The resuscitation and ongoing management of infants born at a GA $<24$ weeks widely vary among countries due to great concerns about the increased number of surviving infants with neurodevelopmental impairments [25, 26]. Our results seem to suggest the use of aggressive management for extremely preterm infants by neonatologists in Japan.

In Japan, echocardiography is routinely performed for hemodynamic management of preterm infants, and this might influence the early detection of PDA, which might explain the decreasing tendency of severe IVH and PVL found in our study [27].

Some of the temporal differences in the antenatal characteristics are interesting. Similar to previously published epidemiologic data $[28,29]$, the prevalence of DM was found to increase, over time, in our study cohort. As 
maternal age is thought to be correlated with DM [30], our data were consistent with those available in the existing literature. Moreover, the current screening and diagnosis of DM, updated in 2010 [31], might also have contributed to the prevalence of DM, over time, in our study cohort. The clinical explanation for our observation in the increase in the prevalence of clinical CAM is unclear. This could possibly be attributed to the fact that the screening and diagnosis of clinical CAM by attending obstetricians, over time, are being conducted in a more careful manner [32]. The prevalence of ANS use in Japan is very low compared to that in other countries; [6] this could be because ANS used to be off-label in Japan until 2009. Considering the advantages of ANS use even for the long-term prognosis of infants born at a GA $<24$ weeks [33], the increased promotion of ANS use in preterm infants might lead to improved mortality outcomes and long-term prognoses, in Japan.

This study has some limitations, the most important being the low follow-up rate ( $48 \%$ at 3 years of age among surviving in-born infants across all institutions, over time). Moreover, infants with follow-up data at 3 years of age had a significantly lower GA and birth weight, and had more morbidities than those without follow-up data (Supplementary Tables 2 and 3). Therefore, in our study, worseaffected infants might have had higher rates of regular hospital follow-ups, which might have led to an overestimation of the long-term outcomes. Although loss to follow-up is actually a common problem in most cohort studies [34-36], concerted efforts must be made to improve the follow-up rate in the NRNJ. Regardless of this, this large cohort study still showed sufficient power in determining the prevalence and correlates of long-term neurodevelopmental outcomes, based on careful interpretation. Second, due to the retrospective design of this study, it might be difficult to determine which of the interventions improved long-term neurodevelopmental outcomes. The high AORs of medical interventions for abnormal long-term outcomes might simply suggest that the most ill infants had received those interventions. For the analysis of the riskadjusted trends, over time, we targeted inborn infants from 38 of the originally participating hospitals in the NRNJ, for the entire study period. These outcomes should be carefully interpreted, as center-related variations inside the network cannot be completely ruled out, as is the case in most cohort studies [9]. We performed multiple testing in the study, wherein the model adjusts for some factors, such as ANS use and cesarean section, that are likely causally related to the changes in the outcomes, over time. However, those changeable factors should be included for the evaluation of the trends in the outcomes, over time, because they are actually important perinatal factors, as indicated by the univariate logistic regression analysis for mortality $[33,37]$.
In conclusion, this large retrospective cohort study determined the trends in mortality and long-term neurodevelopmental outcomes among preterm infants born at a GA $\leq 32$ weeks, over the past decade, in Japan. The trends in terms of the neurodevelopmental outcomes showed a significant downward trend, over time, with the same trend in mortality, suggesting that the increased number of surviving preterm infants were not associated with an increased risk of abnormal neurodevelopmental outcomes. Nutritional support such as intravenous hyperalimentation might be important, and lead to a decreased prevalence of visual impairment, while the early establishment of enteral feeding might lead to improved long-term neurodevelopmental outcomes.

Acknowledgements The authors thank Satoru Shimizu, Tokyo Women's Medical University, for providing excellent advice regarding our data analysis. The authors are indebted to all the infants and their families for their participation in this study. The institutions enrolled in the study of the Neonatal Research Network, Japan, are as follows: Sapporo City General Hospital, Asahikawa Kosei General Hospital, Engaru-Kosei General Hospital, Kushiro Red Cross Hospital, ObihiroKosei General Hospital, Tenshi Hospital, NTT Higashinihon Sapporo Hospital, Nikko Memorial Hospital, Nayoro City General Hospital, Sapporo Medical University, Asahikawa Medical University, Aomori Prefectural Central Hospital, Iwate Medical University, Iwate Prefectural Ofunato Hospital, Iwate Prefectural Kuji Hospital, Iwate Prefectural Ninohe Hospital, Sendai Red Cross Hospital, Akita Red Cross Hospital, Tsuruoka Municipal Shonai Hospital, Yamagata University, Yamagata Prefectural Central Hospital, Fukushima Medical University, Takeda General Hospital, Fukushima National Hospital, Tsukuba University, Tsuchiura Kyodo Hospital, Ibaraki Children's Hospital, Dokkyo Medical University, Jichi Medical University, Ashikaga Red Cross Hospital, Gunma Children's Medical Center, Kiryu Kosei General Hospital, Fuji Heavy Industries Health Insurance Society Ota Memorial Hospital, Gunma University, Saitama Children's Medical Center, Nishisaitama-chuo National Hospital, Saitama Medical University Saitama Medical Center, Kawaguchi Municipal Medical Center, Jichi Medical University Saitama Medical Center, Asahi General Hospital, Chiba Kaihin Municipal Hospital, Kameda Medical Center, Tokyo Women's Medical University Yachiyo Medical Center, Juntendo University Urayasu Hospital, Tokyo Metropolitan Children's Medical Center, Tokyo Women's Medical University, Aiiku Hospital, Nihon University Itabashi Hospital, National Center for Global Health and Medicine, Tokyo Medical University, Teikyo University, Showa University, Japan Red Cross Medical Center, National Center for Child Health and Development, Tokyo Metropolitan Otsuka Hospital, Toho University, Tokyo Metropolitan Bokuto Hospital, Tokyo Jikei Medical University, Tokyo Medical and Dental University, Saint Luku's International Hospital, Juntendo University, Sanikukai Hospital, Katsushika Red Cross Hospital, Yokohama Rosai Hospital, Yokohama City University Medical Center, St. Marianna University School of Medicine Hospital, Kanagawa Children's Medical Center, Tokai University, Kitazato University, Odawara Municipal Hospital, Nippon Medical School Musashi Kosugi Hospital, Saiseikai Yokohamashi Tobu Hospital, National Hospital Organization Yokohama Medical Center, Yamanashi Prefectural Central Hospital, Nagano Children's Hospital, Shinshu University, Iida Municipal Hospital, National Hospital Organization Shinshu Ueda Medical Center, Saku General Hospital, Niigata University, Niigata Prefectural Central Hospital, Niigata Municipal Hospital, Nagaoka Red Cross Hospital, Koseiren Takaoka Hospital, Toyama Prefectural Central 
Hospital, Toyama University, Ishikawa Medical Center for Maternal and Child Health, Kanazawa Medical University, Kanazawa Medical Center, Fukui Prefectural Hospital, Fukui University, Gifu Prefectural General Medical Center, National Hospital Organization Nagara Medical Center, Takayama Red Cross Hospital, Seirei Hamamatsu Hospital, Shizuoka Saiseikai Hospital, Shizuoka Children's Hospital, Hamamatsu Medical University, Numazu Municipal Hospital, Yaizu City Hospital, Fujieda Municipal General Hospital, Nagoya Red Cross Daini Hospital, Nagoya University, Nagoya Red Cross Daiichi Hospital, Toyohashi Municipal Hospital, Nagoya City West Medical Center, Anjo kosei Hospital, Tosei General Hospital, Komaki Municipal Hospital, TOYOTA Memorial Hospital, Okazaki Municipal Hospital, Konan Kosei Hospital, National Mie Central Medical Center, Ise Red Cross Hospital, Yokkaichi Municipal Hospital, Otsu Red Cross Hospital, Shiga University of Medical Science Hospital, Nagahama Red Cross Hospital, Uji Tokushukai Hospital, The Japan Baptist Hospital, Kyoto University, Kyoto Red Cross Daiichi Hospital, National Maizuru Medical Center, Fukuchiyama City Hospital, Kyoto Prefectural University of Medicine Hospital, Kyoto City Hospital, Mitsubishi Kyoto Hospital, Yodogawa Christian Hospital, Osaka Medical Center and Research Institute for Maternal and Child Health, Osaka University, Takatsuki General Hospital, Kansai Medical University, Osaka City General Hospital, Osaka City Sumiyoshi Hospital, Aizenbashi Hospital, Toyonaka Municipal Hospital, National Cerebral and Cardiovascular Center, Kitano Hospital, Saiseikai Suita Hospital, Chifune Hospital, Bellland General Hospital, Rinku General Medical Center, Osaka Red Cross Hospital, Yao Municipal Hospital, Osaka General Medical Center, Osaka City University, Hyogo Prefectural Kobe Children's Hospital, Kobe University, Kakogawa West City Hospital, Saiseikai Hyogoken Hospital, Kobe City Medical Center General Hospital, Hyogo College of Medicine Hospital, Himeji Red Cross Hospital, Toyooka Public Hospital, Hyogo Prefectural Awaji Medical Center, Nara Medical University, Wakayama Medical University, Tottori Prefectural Central Hospital, Tottori University, Shimane Prefectural Central Hospital, Matsue Red Cross Hospital, Kurashiki Central Hospital, Tsuyama Central Hospital, Kawasaki Medical School Hospital, National Hospital Organization Okayama Medical Center, Okayama Red Cross Hospital, Hiroshima City Hiroshima Citizens Hospital, Hiroshima Prefectural Hospital, Hiroshima University, Tsuchiya General Hospital, National Hospital Organization Kure Medical Center, Yamaguchi University, Yamaguchi Grand Medical Center, Tokushima University, Tokushima Municipal Hospital, Kagawa University, National Hospital Organization Kagawa Children's Hospital, Matsuyama Red Cross Hospital, Ehime Prefectural Central Hospital, Kochi Health Science Center, St.Mary's Hospital, National Kyushu Medical Center, Kurume University, Kitakyushu Municipal Medical Center, University of Occupational and Environmental Health, Fukuoka University, Kyushu University, lizuka Hospital, National Hospital Organization Kokura Medical Center, National Hospital Organization Saga Hospital, National Hospital Organization Nagasaki Medical Center, Kumamoto City Hospital, Kumamoto University, Oita Prefectural Hospital, Almeida Memorial Hospital, Nakatsu Municipal Hospital, Miyazaki University, National Hospital Organization Miyakonojo Medical Center, Kagoshima City Hospital, Imakiire General Hospital, Okinawa Prefectural Nanbu Medical Center \& Children's Medical Center, Okinawa Prefectural Chubu Hospital, Naha City Hospital, Okinawa Red Cross Hospital.

Funding No funding was secured for this study.

\section{Compliance with ethical standards}

Conflict of interest The authors declare that they have no conflict of interest.
Open Access This article is licensed under a Creative Commons Attribution 4.0 International License, which permits use, sharing, adaptation, distribution and reproduction in any medium or format, as long as you give appropriate credit to the original author(s) and the source, provide a link to the Creative Commons license, and indicate if changes were made. The images or other third party material in this article are included in the article's Creative Commons license, unless indicated otherwise in a credit line to the material. If material is not included in the article's Creative Commons license and your intended use is not permitted by statutory regulation or exceeds the permitted use, you will need to obtain permission directly from the copyright holder. To view a copy of this license, visit http://creativecommons. org/licenses/by/4.0/.

\section{References}

1. Fanaroff AA, Stoll BJ, Wright LL, Carlo WA, Ehrenkranz RA, Stark AR, et al. Trends in neonatal morbidity and mortality for very low birthweight infants. Am J Obstet Gynecol. 2007;196:147.e1-8.

2. Itabashi K, Horiuchi T, Kusuda S, Kabe K, Itani Y, Nakamura T, et al. Mortality rates for extremely low birth weight infants born in Japan in 2005. Pediatrics. 2009;123:445-50.

3. Shah PS, Sankaran K, Aziz K, Allen AC, Seshia M, Ohlsson A, et al. Outcomes of preterm infants $<29$ weeks gestation over 10 year period in Canada: a cause for concern? J Perinatol. 2012;32:132-8.

4. Horbar JD, Carpenter JH, Badger GJ, Kenny MJ, Soll RF, Morrow KA, et al. Mortality and neonatal morbidity among infants 501 to 1500 grams from 2000 to 2009 . Pediatrics. 2012;129:1019-26.

5. Grisaru-Granovsky S, Reichman B, Lerner-Geva L, Boyko V, Hammerman C, Samueloff A, et al. Population-based trends in mortality and neonatal morbidities among singleton, very preterm, very low birth weight infants over 16 years. Early Hum Dev. 2014;90:821-7.

6. Shah PS, Lui K, Sjors G, Mirea L, Reichman B, Adams M, et al. Neonatal outcomes of very low birth weight and very preterm neonates: an international comparison. J Pediatr. 2016;177:144-52. e6

7. Draper ES, Zeitlin J, Fenton AC, Weber T, Gerrits J, Martens G, et al. Investigating the variations in survival rates for very preterm infants in 10 European regions: the MOSAIC birth cohort. Arch Dis Child Fetal Neonatal Ed. 2009;94:F158-63.

8. Isayama T, Lee SK, Mori R, Kusuda S, Fujimura M, Xiang YY, et al. Comparison of mortality and morbidity of very low birth weight infants between Canada and Japan. Pediatrics. 2012;130:e957-65.

9. Kusuda S, Fujimura M, Sakuma I, Aotani H, Kabe K, Itani Y, et al. Morbidity and mortality of infants with very low birth weight in Japan: center variation. Pediatrics. 2006;118:e1130-8.

10. Rysavy MA, Marlow N, Doyle LW, Tyson JE, Serenius F, Iams JD, et al. Reporting outcomes of extremely preterm births. Pediatrics. 2016. https://doi.org/10.1542/peds.2016-0689.

11. Bax MC. Terminology and classification of cerebral palsy. Dev Med Child Neurol. 1964;6:295-7.

12. Ishii N, Kono Y, Yonemoto N, Kusuda S, Fujimura M. Neonatal Research Network J. Outcomes of infants born at 22 and 23 weeks' gestation. Pediatrics. 2013;132:62-71.

13. Kono Y, Yonemoto N, Kusuda S, Hirano S, Iwata O, Tanaka K, et al. Developmental assessment of VLBW infants at 18 months of age: A comparison study between KSPD and Bayley III. Brain Dev. 2015;38:377-85.

14. Bax MC. Terminology and classification of cerebral palsy. Dev Med Child Neurol. 1964;6:295-7.

15. Jobe AH, Bancalari E. Bronchopulmonary dysplasia. Am J Respir Crit Care Med. 2001;163:1723-9. 
16. Ben-Sira I. An international classification of retinopathy of prematurity. Pediatrics. 1984;74:127-33.

17. Younge N, Goldstein RF, Bann CM, Hintz SR, Patel RM, Smith $\mathrm{PB}$, et al. Survival and neurodevelopmental outcomes among periviable infants. N Engl J Med. 2017;376:617-28.

18. Strommen K, Blakstad EW, Moltu SJ, Almaas AN, Westerberg AC, Amlien IK, et al. Enhanced nutrient supply to very low birth weight infants is associated with improved white matter maturation and head growth. Neonatology. 2015;107:68-75.

19. Stephens BE, Walden RV, Gargus RA, Tucker R, McKinley L, Mance M, et al. First-week protein and energy intakes are associated with 18-month developmental outcomes in extremely low birth weight infants. Pediatrics. 2009;123:1337-43.

20. De Curtis M, Rigo J. The nutrition of preterm infants. Early Hum Dev. 2012;88:S5-S7.

21. Vohr BR, Poindexter BB, Dusick AM, McKinley LT, Wright LL, Langer JC, et al. Beneficial effects of breast milk in the neonatal intensive care unit on the developmental outcome of extremely low birth weight infants at 18 months of age. Pediatrics. 2006;118: e115-23.

22. Engstrom E, Niklasson A, Wikland KA, Ewald U, Hellstrom A. The role of maternal factors, postnatal nutrition, weight gain, and gender in regulation of serum IGF-I among preterm infants. Pediatr Res. 2005;57:605-10.

23. Cayabyab R, Ramanathan R. Retinopathy of prematurity: therapeutic strategies based on pathophysiology. Neonatology. 2016;109:369-76.

24. Hellström A, Engström E, Hård AL, Albertsson-Wikland K, Carlsson B, Niklasson A, et al. Postnatal serum insulin-like growth factor I deficiency is associated with retinopathy of prematurity and other complications of premature birth. Pediatrics. 2003;112:1016-20.

25. Rysavy MA, Li L, Bell EF, Das A, Hintz SR, Stoll BJ, et al. Between-hospital variation in treatment and outcomes in extremely preterm infants. N Engl J Med. 2015;372:1801-11.

26. Marlow N. The elephant in the delivery room. N Engl J Med. 2015;372:1856-7.

27. O’Rourke DJ, El-Khuffash A, Moody C, Walsh K, Molloy EJ. Patent ductus arteriosus evaluation by serial echocardiography in preterm infants. Acta Paediatr. 2008;97:574-8.
28. Jovanovič L, Liang Y, Weng W, Hamilton M, Chen L, Wintfeld $\mathrm{N}$. Trends in the incidence of diabetes, its clinical sequelae, and associated costs in pregnancy. Diabetes Metab Res Rev. 2015;31:707-16.

29. Fadl HE, Simmons D. Trends in diabetes in pregnancy in Sweden 1998-2012. BMJ Open Diabetes Res Care. 2016;4: e000221.

30. Lavery JA, Friedman AM, Keyes KM, Wright JD, Ananth CV. Gestational diabetes in the United States: temporal changes in prevalence rates between 1979 and 2010. BJOG. 2017;124: 804-13.

31. International Association of Diabetes and Pregnancy Study Groups Consensus Panel, Metzger BE, Gabbe SG, Persson B, Buchanan TA, Catalano PA, et al. International association of diabetes and pregnancy study groups recommendations on the diagnosis and classification of hyperglycemia in pregnancy. Diabetes Care. 2010;33:676-82.

32. Fassett MJ, Wing DA, Getahun D. Temporal trends in chorioamnionitis by maternal race/ethnicity and gestational age (19952010). Int J Reprod Med. 2013;2013:906467.

33. Mori R, Kusuda S, Fujimura M, Neonatal Research Network J.. Antenatal corticosteroids promote survival of extremely preterm infants born at 22 to 23 weeks of gestation. J Pediatr. 2011;159:110-4.

34. Tin W, Fritz S, Wariyar U, Hey E. Outcome of very preterm birth: children reviewed with ease at 2 years differ from those followed up with difficulty. Arch Dis Child Fetal Neonatal Ed. 1998;79: F83-87.

35. Castro L, Yolton K, Haberman B, Roberto N, Hansen NI, Ambalavanan N, et al. Bias in reported neurodevelopmental outcomes among extremely low birth weight survivors. Pediatrics. 2004;114:404-10.

36. Fewtrell MS, Kennedy K, Singhal A, Martin RM, Ness A, Hadders-Algra M, et al. How much loss to follow-up is acceptable in long-term randomised trials and prospective studies? Arch Dis Child. 2008;93:458-61.

37. Furukawa S, Sameshima H, Ikenoue T. The impact of cesarean section on neonatal outcome of infants born at 23 weeks of gestation. Early Hum Dev. 2014;90:113-8. 